\title{
The use of occlusion to resolve ambiguity in parallel projections
}

\author{
MYRON L. BRAUNSTEIN, GEORGE J. ANDERSEN, and DAVID M. RIEFER \\ University of California, Irvine, California 92717
}

\begin{abstract}
Previous research has shown that the three-dimensional structure of an object usually can be perceived when viewing a parallel projection of the object rotating in depth. Accurate judgments of direction of rotation, however, have been found only with polar projections. The present study demonstrated that accurate direction judgments can occur with parallel projections if occlusion is included in the displays. The stimuli were parallel projections of pentagonal texture elements on the surface of a rotating sphere. In one condition, the elements were occluded as they rounded the edge of an opaque sphere. In another condition, elements on the far surface of a transparent sphere were occluded by elements on the near surface. Accuracy of direction judgments was consistently high in the first condition and increased monotonically with element size in the second condition, from chance to over $80 \%$ correct. The relationship of these results to the general issue of perceptually combining structure in depth information from one source with relative distance information from another source is discussed.
\end{abstract}

A two-dimensional projection of a surface moving in three-dimensional space can produce a compelling impression of motion in depth, even when the projection contains no information about the relative distances of parts of the surface from the observer. This result has been obtained in a number of studies using parallel projections of rotating objects (Braunstein, 1977a, 1977b; Petersik, 1980a; Ullman, 1979; see Braunstein, 1976, for a review of earlier research). In earlier research, parallel projections were approximated by projecting shadows of real objects onto a translucent screen, using a distant light source. More recent studies have used computergenerated displays in which a hypothetical threedimensional object is mathematically projected onto a plane surface using an infinitely distant projection point. (Parallel projections can be approximated in direct vision of distant objects, especially in telescopic views.) In almost all of the research using parallel projections, real or simulated objects were displayed rotating about an axis (usually a vertical axis) perpendicular to the line of sight. Direction of rotation judgments were often used as a measure of subjects' ability to perceive relative distance in the rotating configurations. As expected, these judgments did not exceed chance levels for parallel projections.

Accurate direction of rotation judgments can be obtained with polar projections, provided that the projection point is close enough to the rotating sur-

This research was supported by a University of California Faculty Research Grant to the first author. The second author received support from a Research Assistantship awarded by the University of California Focused Research Program in Perception and Higher Mental Processes. face. (The projection point distance simulates the eye distance in direct vision.) The relationship between projection point distance and accuracy depends on the shape of the rotating figure (Braunstein \& Payne, 1968). The geometrical effects of projection point distance on a projected image are generally referred to as perspective effects. Johansson (1974) and Gibson (1957) have stressed the role of perspective in depth perception, and the role of perspective in disambiguating relative distance in rotating surfaces supports their viewpoints. ${ }^{1}$

Combining the results with parallel projections of rotating surfaces with those obtained with polar projections leads to the following general conclusion: An observer can perceive the overall structure of a surface from the information available in a parallel projection, and can use the additional information available in a polar projection to determine which part of the surface is closer and which is more distant (or to determine the direction of rotation). It should not be concluded, however, that polar perspective is the only source of information available to the monocular observer that can disambiguate relative depth in a parallel projection. Laboratory research on the perception of rotary motion has been limited to shadow projections or computer-generated displays with transparent texture elements. In the visual environment outside of the laboratory, on the other hand, shadows and transparent surfaces are relatively infrequent. As an observer moves among nontransparent surfaces, or as these surfaces move relative to an observer, kinetic occlusion is likely to be a major source of relative distance information (Gibson, Kaplan, Reynolds, \& Wheeler, 1969). Kinetic 
occlusion refers to the appearance and disappearance from view of texture elements on a surface that is moving behind another surface, or changing its orientation relative to the observer. Unlike static interposition, kinetic occlusion is not limited to a class of recognizable contour interruptions, but can be based entirely on dynamic information (Rhodes, 1980).

Kaplan (1969) studied the effects of kinetic occlusion on the perception of the relative distances of two surfaces translating horizontally. Texture elements were added or deleted on one or both sides of an unmarked vertical edge separating the surfaces. If one region showed accretion or deletion of texture elements, it was reported to be the more distant surface. If both regions showed accretion or deletion of texture elements, the region showing the greater rate of texture gain or loss was reported to be the more distant surface. Rhodes (1980) extended Kaplan's study to include up to three translating regions, as well as brightness and texture density variations. These studies do not bear directly on the question of whether occlusion can add relative distance information to the structure in depth information available in parallel projections. Parallel projections of translatory motion do not provide structure in depth information (Braunstein, 1976; Johansson, 1977). Polar perspective, as reflected by the relative velocities of the moving fields, did not produce consistent relative depth judgments in Kaplan's or Rhodes' experiments. This is to be expected, as accurate relative depth judgments based on motion parallax have been found only with a moving observer or display screen (Rogers \& Graham, 1979) or with a continuous gradient of velocities (Braunstein \& Andersen, 1981).

The purpose of the present study is to demonstrate that kinetic occlusion can disambiguate depth relationships in a parallel projection and thus, in effect, can substitute for polar perspective in providing an unambiguous perception of a rotating surface. It is generally recognized that depth can be perceived, up to a reflection about the line of sight, from a parallel projection and that the remaining ambiguity is removed in a polar projection (Braunstein, 1976; Ullman, 1979). The present study tests the more general proposition that subjects can combine the information about internal depth available in a parallel projection with relative depth information from another source to perceive both structure in depth and relative distance in a rotating surface.

Two types of kinetic occlusion will be considered. The first is the appearance and disappearance from view of texture elements on an opaque surface during changes in the orientation of the surface relative to an observer or as one surface moves behind another surface (Kaplan, 1969; Rhodes, 1980). This will be referred to as edge occlusion. A natural ex- ample of edge occlusion is the gradual appearance of the ground plane below a cliff as an observer on the cliff approaches the edge (Gibson, 1979). In the present study, edge occlusion will be represented by the appearance and disappearance from view of texture elements on the surface of an unseen sphere as the sphere is rotated about a vertical axis.

In the second type of kinetic occlusion, the texture elements do not lie on an opaque surface. They may lie on a transparent surface or not lie on a surface. In this type of occlusion, individual near elements can occlude all or part of individual distant elements. This will be referred to as element occlusion. A natural example of element occlusion is the gradual covering and uncovering, in the retinal projection, of one tree by another as an observer moves about in a thick wood. In the present study, element occlusion will be represented by the occlusion of the more distant elements on the surface of a transparent sphere by the closer elements, as the sphere rotates about a vertical axis. The distinction between edge occlusion and element occlusion is somewhat arbitrary, especially in a natural environment, but should be useful in the systematic study of the effectiveness of occlusion in depth perception.

In addition to type of occlusion (edge vs. element), the present study included two independent variables that were expected to determine the effectiveness of occlusion information. The first, texture element size, was expected to have its primary effect on element occlusion. With the number of elements held constant, the number of visible occlusions of a back element by a front element is, of course, a function of element size.

The second additional variable was visibility of the edge of the sphere. The texture elements in the edgeocclusion displays undergo their maximum horizontal contraction just before disappearing from view. It was expected that the perception of the elements as moving around the edge of the sphere would be enhanced when this maximum contraction was visible, and that the direction of rotation judgments would be less accurate when the edge of the sphere was concealed.

\section{METHOD}

\section{Subjects}

The subjects were 34 students in lower division psychology courses at the University of California, Irvine, who received extra credit for their participation. Vision of at least 20/40 (Snellen eye chart) was required in the eye used in the experiment. Data from three subjects were not used because of failure to follow instructions. Data from one subject was not used because of problems with the apparatus.

\section{Design}

Four independent variables were examined: type of occlusion (edge or element), size of the texture elements (five levels), edge 
visibility (three levels), and direction of rotation. Type of occlision, element size, and direction of rotation were run as withinsubjects variables. Edge visibility was run between subjects, with 10 subjects run at each level.

\section{Stimuli}

The stimuli were 207.5 -sec (120-frame) computer-generated 16-mm motion picture sequences. The sequences were generated one frame at a time on a Tektronix $\mathbf{4 0 1 0}$ display scope, recorded on Kodak Plus-X film using an Automax camera, and printed on high-contrast film. The 20 sequences were randomly arranged on the film with a 7.5-sec (120-frame) ISI. Each sequence displayed a parallel projection of texture elements located on the surface of a sphere rotating about a vertical axis. The texture elements were irregular pentagons. (The pentagon shape was selected to reduce the likelihood of false slant impressions that might occur with irregular triangles or quadrilaterals.)

The 50 pentagons were located on the surface of the sphere in the following manner: First, 50 points were arbitrarily selected on the surface of a sphere so as to appear approximately evenly distributed when the sphere was viewed from various angles. The point locations were then adjusted so that no two adjacent pentagons of the largest size would overlap. For each of the 20 displays, a pentagon was drawn at each of the 50 locations by a random procedure, with the constraint that an angle formed by connecting the center to any two adjacent vertices would be between .35 and 2.09 radians (to avoid extreme shapes).

There were five element sizes. The smallest was comparable to the "dots" used in previous studies, in that it was completely filled. The total proportion of the surface area covered by the 50 elements was $.00005, .0073, .035, .106$, and .25 , for the five element sizes.

In generating the edge-occlusion displays, the surface of the sphere was treated as opaque and the texture elements appeared and disappeared from view as they rounded the edge of the sphere. Approximately half of the texture elements were visible on a frame of the motion picture. In generating the element-occlusion displays, the surface was treated as transparent but the elements were treated as opaque. All elements were visible on every frame, but some of the elements on the more distant hemisphere were partially occluded on the closer hemisphere. There was no elementocclusion information available in the displays with the smallest size texture elements, as the projection of two filled dots coming together is the same regardless of which dot is nearer (as in the case of shadow projections). The mean number of occlusions per frame for the four larger element size displays were approximately $1.3,4.7,11.8$, and 25.9 , respectively. Figure 1 shows the 10 combinations of element size and type of occlusion. Each sequence consisted of 180 -deg clockwise or counterclockwise rotation of the sphere.

\section{Apparatus}

A 16-mm projector (L-W Athena) was used to present the film. The film was projected through a glass window onto a translucent screen (Polacoat) in a separate room. The pentagon and background luminances at the screen were 3.0 and $.02 \mathrm{~cd} / \mathrm{m}^{2}$, respectively. A . 4 neutral density filter was located in the viewing tube to enhance the perception of the background as totally dark.

The subjects viewed the screen monocularly from the opposite side at a distance of $1.8 \mathrm{~m}$ through a tube that restricted the field of view to a circular region. In one condition, the edge of the projected sphere extended outside the limits of the viewing area. In a second condition, the edge of the sphere was aligned with the edge of the viewing area. In a third condition, the viewing area was larger than the projected sphere. The field of view was 14, 17 , or $20 \mathrm{deg}$ (diameter in visual angle), respectively, in the three edge-visibility conditions. The diameter of the projected sphere was $17 \mathrm{deg}$ in all conditions.

A 15-cm-diam glass sphere with white dots unsystematically positioned on the surface served as a demonstration model. An 8-rpm reversible motor was attached to the vertical axis of the sphere.

The response device consisted of a 50 -mm-diam spherical knob mounted on a vertical shaft. Turning the knob clockwise or counterclock wise triggered one of two microswitches which turned on one of two lights at the experimenter's console.

\section{Procedure}

The subjects were run individually. They were instructed to indicate by turning the response device clockwise or counterclockwise,
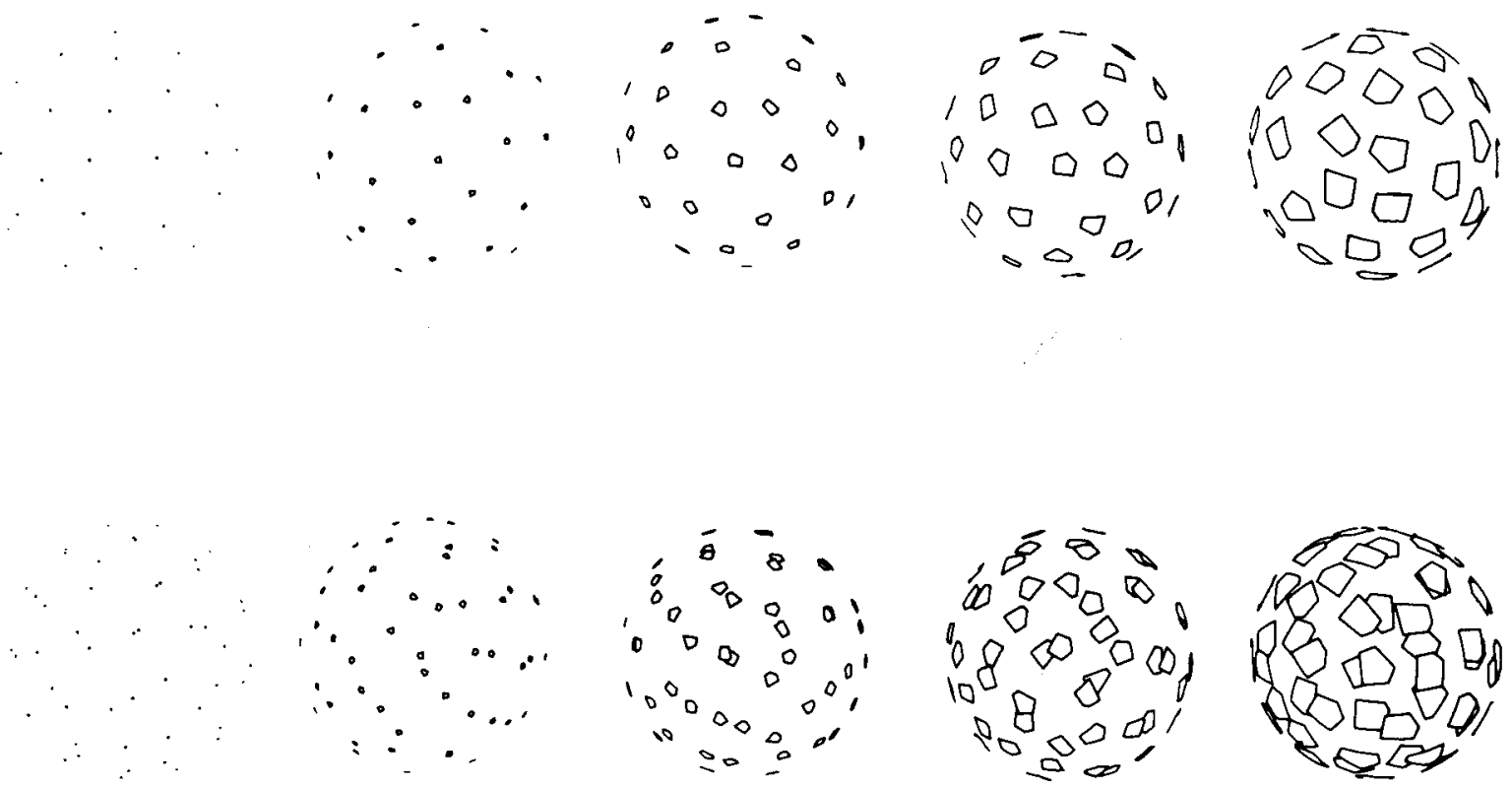

Figure 1. Single frames from the edge-occlusion (top) and element-occlusion (bottom) sequences for each level of textureelement size. (The stimulus film showed white figures on a black background.) 
the direction of rotation of the sphere viewed through the tube as soon as each display disappeared. The two directions of rotation were demonstrated using the glass display model, and the subject practiced the response procedure. The room was then made totally dark, and the film was started.

A different order of stimulus presentation was obtained for each subject in a given edge visibility condition by running the film in either the forward or reverse direction with five equally spaced starting positions used in each direction. (The same 10 orders were used in each of the three edge-visibility conditions.) The first five displays served as practice trials and were repeated at the end of the first block of 20 trials. The practice trials were followed by the first block of 20 sequences. The 20 sequences were then repeated in reverse order, with the direction of rotation in each sequence reversed, and the subject continued to give directionof-rotation responses. The subject was then instructed to judge the coherence or rigidity of each display on a 4-point scale. A rating of 1 was to be used for nonrigid displays, for example, displays that appeared to consist of two or more independently moving surfaces. A rating of 4 was to be used for completely rigid displays. Ratings of 2 and 3 were to represent "somewhat rigid displays" and "mostly rigid displays," respectively. The subjects were told to give their coherence ratings verbally as soon as each display disappeared. The 20 displays were presented in the same order as the first block of 20 trials. At the end of the session, the subject was asked a series of questions about the displays, beginning with, "What did you think you were looking at?"

\section{RESULTS}

\section{Accuracy}

Each subject made two direction-of-rotation responses to each stimulus sequence, under one of the three edge-visibility conditions. Accuracy was defined as the frequency $(0,1$, or 2$)$ with which these responses matched the simulated direction of rotation. (A simulated direction was assigned to all sequences, although the element occlusion sequences with the smallest size elements did not contain information from which direction of rotation could be veridically determined.) The effects of the four independent variables (viewing area, type of occlu-

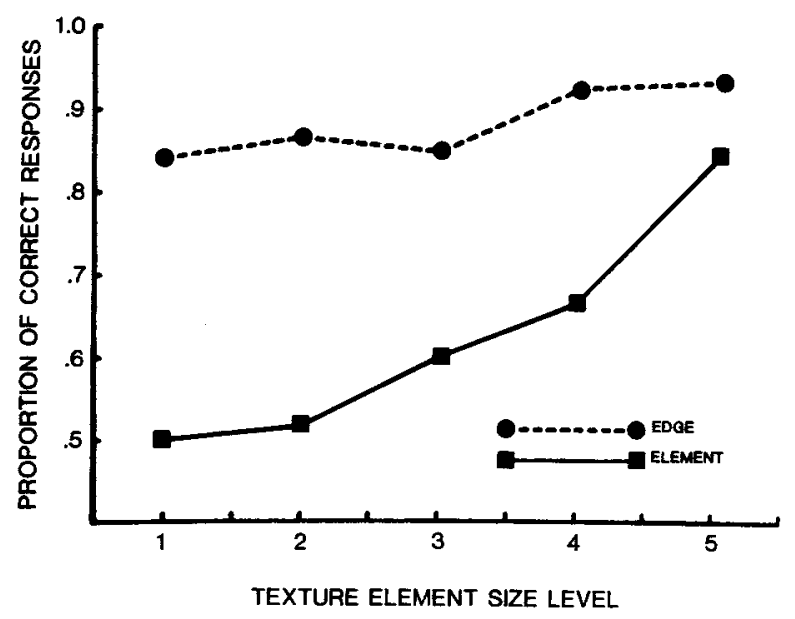

Figure 2. Effects of texture-element size on proportion of correct responses for edge and element occlusion. sion, element size, and direction of rotation) were examined in an analysis of variance. The main effects of occlusion type $[F(1,27)=54.6]$ and of element size $[F(4,108)=22.9]$ and the interaction of type and size $[F(4,108)=5.57]$ were significant (ps $<$ $.01)$. Figure 2 shows the effects of type and size on the accuracy of direction judgments. Accuracy increased from chance at the smallest size level to over $80 \%$ correct at the largest size level, for the element-occlusion displays. For the edge-occlusion displays, accuracy exceeded $\mathbf{8 0 \%}$ correct for all size levels, and exceeded $90 \%$ with the largest texture elements. The main effects of edge visibility $[F(2,27)$ $<1]$ and of direction of rotation $[F(1,27)=3.27]$ were not significant. There were no other significant interactions.

\section{Coherence}

The coherence rating given by each subject to each display (1-4) were subjected to an analysis of variance. The independent variables were the same as in the accuracy analysis. The main effect of type of occlusion was significant $[F(1,27)=16.5, p<.01]$. The mean coherence ratings for edge and element occlusion were 3.24 and 2.76 . The lower coherence rating with element occlusion is consistent with the informal observation that these displays sometimes took on the appearance of two hemispheres with the same curvature, moving in opposite directions. There was a significant interaction between type of occlusion and element size $[F(4,108)=3.18, p<.05]$. Coherence ratings increased with element size for the edgeocclusion displays but not for the element-occlusion displays.

The main effect of direction of rotation was significant $[\mathrm{F}(1,27)=7.72, \mathrm{p}<.01]$. Counterclockwise rotations were rated as more coherent than clockwise rotations (3.11 vs. 2.89 ). There were significant interactions between type and direction $[\mathrm{F}(1,27)=5.58$, $\mathrm{p}<.05]$ and between type, size, and direction $[\mathrm{F}(4,108)$ $=7.63, \mathrm{p}<.01]$. The effect of direction of rotation on coherence ratings (higher ratings for counterclockwise stimuli) was associated with edge occlusion rather than element occlusion, and was more pronounced with the larger texture elements. The effects of occlusion type, element size, and direction of rotation on coherence ratings are shown in Figure 3.

The main effects of viewing area $[F(2,27)=1.10]$ and of element size $[F(4,108)=2.24]$ were not significant. There were no other significant interactions.

\section{Debriefing}

In response to the debriefing questions, 25 of the 30 subjects stated that they believed they were watching a three-dimensional object through the viewing tube. The remaining 5 subjects stated that they believed they were watching a film or other twodimensional projection. 


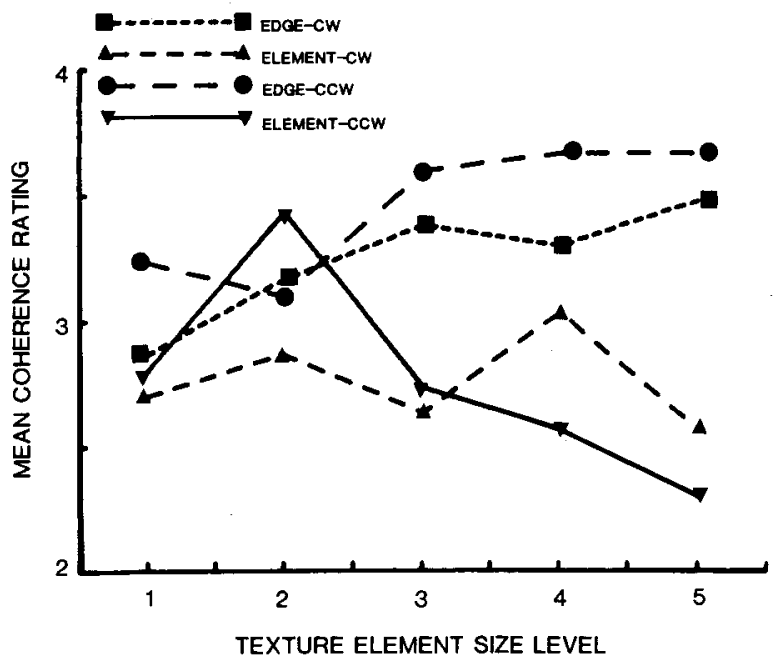

Figure 3. Effects of texture-element size on coherence ratings for edge and element occlusion, displayed with clockwise or counterclockwise rotation.

\section{DISCUSSION}

The accuracy of the direction-of-rotation judgments found for most of the stimulus conditions provides support for the hypothesis that observers can combine structure in depth information from one source with relative distance information from another source. In the present study, structure in depth information was available in the parallel projections of the rotating patterns. Previous research has established the ability of subjects to perceive structure in depth of parallel projections of rotating dot and line patterns (Braunstein, 1966, 1977a, 1977b; Petersik, 1979, 1980a, 1980b; Ullman, 1979). The sinusoidal waveform of the projected motion has been shown to be a sufficient stimulus for the perception of this motion as representing rotation in depth (Braunstein, 1977a). Some structural information also may have been provided by the curved lines constituting the pentagons in the present study. This is especially noticeable with the larger pentagon sizes (see Figure 1) and may account for the increase in coherence ratings found with increased element size for the edge-occlusion displays.

In previous research in which both structure in depth and relative distance information was provided for rotating patterns, the source of relative distance information was polar perspective. The present study demonstrates that this information also can be provided by occlusion. Comparable levels of accuracy in direction of rotation judgments can be achieved from the two alternative sources of information. Braunstein (1977b) found mean proportions of correct responses if .75 and .90 for spheres 39.7 and $9.6 \mathrm{deg}$ in diameter, filled with 1,000 randomly positioned dots, with a projection distance of three radii. The mean accuracy in the present study with the smallest texture-element size stimulus and with edge occlusion rather than polar perspective was .84 . This comparison suggests only that comparable values are obtainable. Either source of information can produce a wide range of accuracy values as projection point distance is varied in one case or the number and size of occluded elements is varied in the other.

Two conclusions can be drawn from the effectiveness of occlusion in providing relative depth information in parallel projections: First, there are limits to the generality of the concept that depth is perceived in dynamic displays by a perceptual process that reverses the mathematics of projective geometry (Johansson, 1974). Johansson (1977) has noted that the reverse perspective model must be qualified by the finding of "the kinetic depth effect" with parallel projections of rotating line patterns. Although polar perspective is an important source of relative distance information, the present results demonstrate that relative distance, as well as structure in depth, can be perceived in the absence of polar perspective. A second conclusion is that it may be useful to classify sources of depth information according to whether they provide information about structure in depth, relative distance, or both. Some sources, such as the sinusoidal waveform of the projected motion, provide only structural information. Others, such as occlusion, appear to provide only relative distance information (Kaplan, 1969). Still other sources, notably polar perspective, provide both types of information (Braunstein, 1966, 1977a). The combining of structural information with relative distance information may be an important aspect of the perceptual organization of the three-dimensional environment.

It is not surprising that accurate judgments of relative distance can be made on the basis of dynamic occlusion. Gibson (1979) has vividly described the role of occlusion in the perception of the natural environment. Occlusion, however, has not always been separated from other information about depth and distance that becomes available when an observer is in motion. A notable example of the failure to recognize the potential effects of dynamic occlusion is found in a statement by Helmholtz $(1925 / 1962$, pp. 295-296) that is used as a classic description of the effects of motion parallax: "Suppose, for instance, that a person is standing still in a thick wood, where it is impossible for him to distinguish, except vaguely and roughly, in the mass of foliage and branches all around him what belongs to one tree and what to another or how far apart the separate trees are, etc. But the moment he begins to move forward, everything disentangles itself and immediately he gets an apperception of the material contents of the woods and their relations to each other in space, just as if he were looking at a good stereoscopic view of it." Motion parallax undoubtedly would play 
an important role in this situation. Rogers and Graham (1979) found that the parallax produced by the observer's head movements provides both structure in depth and relative distance information. However, it is also clear that a continuous covering and uncovering of more distant surfaces by nearer surfaces will occur as the observer walks through the type of environment described by Helmholtz. The work of Kaplan (1969) and Rhodes (1980) with translatory motion and the present results with rotary motion demonstrate that the human observer is able to use these changes in the perception of relative distance.

In interpreting the results of the present experiment, the question might be raised of whether subjects perceived the displays as rigid three-dimensional configurations rotating in a particular direction or whether they had made intellectual judgments about the direction of rotation. This question cannot be answered directly, but there are several reasons to believe that the effect was perceptual and not cognitive. First, the high proportion (.83) of subjects who stated that they believed they were watching actual threedimensional objects supports the overall realism of the stimuli. The high coherence ratings, averaging 3.0 ("mostly rigid") across all stimuli, provides a further indication that the stimuli appeared to be three-dimensional, with all elements rotating in the same direction in most cases. Finally, the gradual increase in accuracy with element size in the elementocclusion displays suggests that the subjects were not merely noting instances of occlusion as a strategy in determining direction of rotation. A single occlusion (together with an observation of whether the occluding element was moving left or right) would have been sufficient to reveal the direction of rotation, yet at the intermediate element size level the proportion of correct responses was only .6 , with an average of almost five occlusions presented on each frame.

The expected effect of edge visibility on the accuracy of direction-of-rotation judgments was not found. Concealing the edge of the sphere did not reduce the accuracy of these judgments. Instead, both accuracy and coherence ratings appear to have reached ceiling levels in the edge-occlusion displays, leaving no room for a further effect of edge visibility. The significant main effect and interactions involving displayed direction of rotation for the coherence ratings were also unexpected. No relationship could be found between the higher coherence ratings given to counterclockwise rotations and the accuracy of direction judgments. No explanation can be offered at this time for the effect of displayed direction of rotation on the coherence ratings.

There are many directions that would be worth pursuing in future research on the role of occlusion in depth perception. The following three research problems provide examples that range from extensions of the present experiment to the exploration of the role of occlusion in another area of motion perception. First, it would be of interest to further separate kinetic occlusion from static interposition effects in the perception of direction of rotation for parallel projections displaying element occlusion. In the present experiment, the individual frames of the element-occlusion stimuli, with the exception of those produced with the smallest element size, displayed static occlusion (interposition) (see Figure 1). It is possible that this static information contributed to the accuracy of the direction-of-rotation judgments. (This possibility does not apply to the edgeocclusion stimuli, which did not display static occlusion.) With the use of more complex stimulus materials, it should be possible in future research to study the effects of kinetic element occlusion without providing static occlusion in individual frames or in momentary views of the stimulus displays.

A second area for future research is the relationship between occlusion and perspective in the perception of relative distance. Although the present results demonstrated that occlusion can serve the same function as perspective in providing direction-of-rotation information, this does not imply that the two sources of information are equivalent in other respects. As noted above, perspective can provide both structure in depth and relative distance information, but occlusion appears to provide only the latter. Occlusion may have a special status as a source of relative distance information, because it is especially resistant to reversal. Displays that include perspective effects can often be reversed (e.g., a wire cube can be seen as a truncated pyramid). Under some circumstances, depth reversal can occur even when veridical relative distance information is available from binocular disparity (Yellott \& Kaiwi, 1979). Occlusion, if it is generally more resistant to reversal, may be important in providing stability in the perception of relative distance. It is expected that when occlusion and perspective provide conflicting information about direction of rotation, perceived direction will be determined exclusively by occlusion.

The third area for future research is the role of occlusion in the perception of egomotion. Dichgans and Brandt (1978) have suggested that motion in the "depth periphery," as well as motion in the retinal periphery, determines the perception of egomotion. As a reliable indicator of relative distance, kinetic occlusion may be of special importance in defining the depth periphery, and may therefore play a major role in the perception of egomotion.

\section{REFERENCES}

Braunstein, M. L. Sensitivity of the observer to transformations of the visual field. Journal of Experimental Psychology, 1966, 72, 683-687.

Braunste IN, M. L. Depth perception through motion. New York: Academic Press, 1976.

Braunstein, M. L. Minimal conditions for the perception of 
rotary motion. Scandinavian Journal of Psychology, 1977, 18 , 216-223. (a)

Braunstein, M. L. Perceived direction of rotation of simulated three-dimensional patterns. Perception \& Psychophysics, 1977. 21, 553-557. (b)

Braunstein, M. L., \& Andersen, G. J. Velocity gradients and relative depth perception. Perception \& Psychophysics, 1981, 29, 145-155.

Braunstein, M. L., \& Payne, J. W. Perspective and the rotating trapezoid. Journal of the Optical Society of America, 1968, 58, 399-403.

Dichgans, J., \& Brandt, Th. Visual-vestibular interactions and motion perception. In R. Held, H. W. Leibowitz, \& H. L. Teuber (Eds.), Handbook of sensory physiology (Vol. 8). Berlin: Springer, 1978.

Gibson, J. J. Optical motions and transformations as stimuli for visual perception. Psychological Review, 1957, 64, 288-295.

GiBson, J. J. The ecological approach to visual perception. Boston: Houghton Mifflin, 1979.

Gibson, J. J., Kaplan, G. A., Reynolds, H. N., JR., \& WheEler, $K$. The change from visible to invisible: A study of optical transitions. Perception \& Psychophysics, 1969, 5, 113-116.

Gogel, W. C. The organization of perceived space: I. Perceptual interactions. Psychologische Forschung, 1973, 36, 195-221.

Helmholtz, H. Physiological optics (Vol. 3) (J. P. Southall, Ed. and trans.). New York: Dover, 1962. (Originally published, Optical Society of America, 1925.)

Jor ansson, G. Projective transformations as determining visual space perception. In R. B. McLeod \& H. L. Pick (Eds.), Perception: Essays in honor of J. J. Gibson. Ithaca, N.Y: Cornell University Press, 1974.

Johansson, G. Spatial constancy and motion in visual perception. In W. Epstein (Ed.), Stability and constancy in visual perception: Mechanisms and processes. New York: Wiley, 1977.

Kaplan, G. A. Kinetic disruption of optical texture: The perception of depth at an edge. Perception \& Psychophysics, 1969, 6, 193-198.

Petersik, J. T. Three-dimensional object constancy: Coherence of a simulated rotating sphere in noise. Perception \& Psychophysics, 1979, 25, 328-335.
Petersik, J. T. Rotation judgments and depth judgments: Separate or dependent processes. Perception \& Psychophysics, $1980,27,588-590$. (a)

Petersik, J. T. The effects of spatial and temporal factors on the perception of stroboscopic rotation simulations. Perception, 1980, 9, 271-283. (b)

RHODES, J. W. The effect of multiple depth cues in the perception of occlusion. Unpublished doctoral dissertation, University of Tennessee, Knoxville, 1980.

Rogers, B., \& Graham, M. Motion parallax as an independent cue for depth perception. Perception, 1979, 8, 125-134.

Ullman, S. The interpretation of visual motion. Cambridge, Mass: M.I.T. Press, 1979.

YellotT, J. I., JR., \& Kaiw1, J. L. Depth inversion despite stereopsis: The appearance of random-dot stereograms on surfaces seen in reverse perspective. Perception, 1979, 8, 135-142.

\section{NOTE}

1. "Structure in depth" will be used to refer to depth relationships within a displayed or simulated object, without regard to the location of the observer's viewpoint. Although the concept of perceived structure implies accurate perception of the shape of an object, most studies of perceived structure in parallel projections have not included formal determinations of the accuracy with which an observer can discriminate among alternative threedimensional shapes. Instead, perceived structure usually has been inferred from judgments of whether an object appeared twodimensional or three-dimensional and whether it appeared coherent.

"Relative distance" will be used to describe the order of points or surfaces in distance from the observer, that is, which is closer and which is more distant. Egocentric or absolute distances (Gogel, 1973 ) will not be considered in the present paper. The projections that were displayed can represent a rotating sphere at any distance from the observer, provided that the relationship between size and distance is maintained.

(Manuscript received August 31, 1981; revision accepted for publication November 17, 1981.) 